\title{
New cases of unusual polarimetric behavior in asteroids ${ }^{\star}$
}

\author{
R. Gil-Hutton ${ }^{1,2}$, V. Mesa ${ }^{2}$, A. Cellino ${ }^{3}$, P. Bendjoya ${ }^{4}$, L. Peñaloza ${ }^{2}$, and F. Lovos $^{2}$ \\ ${ }^{1}$ Complejo Astronómico El Leoncito - CONICET, Av. España 1512 sur, J5402DSP San Juan, Argentina \\ e-mail: rgilhutton@casleo.gov.ar \\ 2 Universidad Nacional de San Juan, J. I. de la Roza 590 oeste, 5400 Rivadavia, San Juan, Argentina \\ 3 INAF, Osservatorio Astronomico di Torino, 10025 Pino Torinese (TO), Italy \\ ${ }^{4}$ Laboratoire Fizeau, UMR 6525 Université Nice Sophia Antipolis, Observatoire de la Côte d'Azur, CNRS, \\ 06108 Nice Cedex 2, France
}

Received 30 October 2007 / Accepted 5 February 2008

ABSTRACT

\begin{abstract}
Aims. Results of different polarimetric campaigns at Complejo Astronómico El Leoncito (Casleo), San Juan, Argentina are presented. The aim of these campaigns was to search for objects exhibiting anomalous polarimetric properties, similar to those shown by the Ld-class asteroid (234) Barbara, among members of the same or similar taxonomic classes.

Methods. The data have been obtained with Torino and CASPROF polarimeters at the $2.15 \mathrm{~m}$ telescope. The Torino polarimeter is an instrument that allows simultaneous measurement of polarization in five different bands, and CASPROF polarimeter is a two-hole aperture polarimeter with rapid modulation.

Results. The campaigns began in 2005, and we found four new asteroids with Barbara-like polarimetric properties: the L-class objects (172) Baucis, (236) Honoria and (980) Anacostia, and the K-class asteroid (679) Pax. The polarimetric properties of the phase-polarization curves of these objects may be produced by a mixture of high-and low-albedo particles in their regolith as a result of the fragmentation of a substrate that is spectrally analog to the CO3/CV3 chondrites.
\end{abstract}

Key words. solar system: general - minor planets, asteroids - polarization

\section{Introduction}

When unpolarized light is scattered by a rough surface it becomes partially polarized. The polarization produced is usually found to be linear with its azimuth either normal or parallel to the scattering plane, which in the Solar System is the plane containing the asteroid, the Sun, and the Earth at the epoch of observation. In polarimetry, the results of observations are usually expressed using the parameter $P_{r}=\left(I_{\perp}-I_{\|}\right) /\left(I_{\perp}+I_{\|}\right)$, where $I_{\perp}$ and $I_{\|}$are the intensities of the scattered light polarized along the planes perpendicular and parallel to the scattering plane, respectively.

$P_{r}$ is found to change with the angle between the incident and observation rays, usually known as the phase angle, $\alpha$. A plot of $P_{r}$ against $\alpha$ is known to produce a characteristic curve described by some parameters whose measured values are found to be diagnostic of the overall texture and optical properties of the surface. For phase angles $\lesssim 20^{\circ}, P_{r}$ turns out to be negative, reaching a minimum of polarization, $P_{\min }$, at phase angles $\approx 10^{\circ}$. This general behavior characterizes, with some minor differences (Belskaya et al. 2005) depending on the taxonomic class, all asteroids observed so far. Beyond $\approx 20^{\circ}$ of phase, the polarization changes sign at the inversion angle, $\alpha_{0}$, and becomes positive. The existence of a branch of negative polarization exhibited by atmosphereless Solar System bodies is a well-known fact, which is generally explained in terms of the occurrence

^ Based on observations carried out at the Complejo Astronómico El Leoncito, operated under agreement between the Consejo Nacional de Investigaciones Científicas y Técnicas de la República Argentina and the National Universities of La Plata, Córdoba, and San Juan. coherent backscattering phenomena (Muinonen et al. 2002b, 2007; Tyynelä et al. 2007).

Laboratory measurements on a variety of rocks, meteorites, lunar, and artificial samples, both solid and pulverized, have produced empirical relationships between the polarimetric parameters that can be understood in terms of simple physical processes (Geake \& Dollfus 1986, and references therein). It has been demonstrated experimentally that the polarization parameters $P_{\min }$ and $\alpha_{0}$ are related to the surface texture. Laboratory measurements on bare chips or large fragments of rocks, from the Moon or Earth, are confined to values around $\left|P_{\min }\right|=0.5 \%$ and $\alpha_{0}=13-14^{\circ}$, whereas very finely divided silicaceous powders and lunar fines are grouped around $\left|P_{\min }\right|=1.0 \%$ and $\alpha_{0}=22-23^{\circ}$. Pulverized rocks with grain sizes between 30 and $300 \mu \mathrm{m}$ come in between these two domains. The discrimination is clear, and such plots have been used for the remote analysis of microtextures on the surface of planetary bodies.

Planetary surface analysis by telescopic photopolarimetry and surface landings on the Moon and Mars have already demonstrated that all the atmosphereless planetary objects in the Solar System are subject to the formation on their surfaces of a thick layer of small grains and debris. This layer, denominated the regolith, is produced by meteoritic impacts which accumulate a layer of comminuted fragments, periodically gardened by new impacts that produce further ejecta. For impacts on lunar-sized objects with average impact velocities of several tens of kilometers per second, the cumulative effect of impacts produces a thick layer of $10 \mu \mathrm{m}$ size grains (Gault et al. 1974; Langevin \& Arnold 1977).

In the case of the asteroids the situation is different. For the larger objects, polarimetry indicates a regolithic layer made up 
of a broad mixture of particle sizes, mainly $>50 \mu \mathrm{m}$, but mixed and partially-coated with small particles of $\leq 10 \mu \mathrm{m}$ (Le Bertre $\&$ Zellner 1980). The depletion of small grains, compared to the Moon, results from the escape velocity several times lower for these objects. For the smaller asteroids, the polarimetric signature is consistent with a coarse regolith still more depleted in the smaller particles. This depletion can be easily justified, taking into account that small ejecta from impact events on asteroid surfaces usually achieve speeds well above the escape velocity of their parent body (O'Keefe \& Ahrens 1977).

In principle, therefore, one could expect that the polarimetric properties of asteroids of different sizes and compositions might be quite complex and heterogeneous. Objects of smaller sizes are expected to be increasingly depleted in small regolith particles, and the possible presence of albedo and texture variegation of the surface, could be expected to produce further complications. It is true, however, that so far the observed polarimetric behavior of a large variety of asteroids of different sizes and belonging to different taxonomic classes has been quite homogeneous. The phase-polarization curves displayed by the objects tend to have a uniform general morphology, apart from some variation in some of the features of these curves (depth of the maximum negative polarization; slope of the linear trend close to the inversion angle), that are long known to be correlated with the surface albedo. As an example, the phase-polarization curve of the very small, near-Earth asteroid (25143) Itokawa, measured by Cellino et al. (2005a), allowed them to derive an albedo value for this object in agreement with the result of the subsequent in situ measurements by the Hayabusa space probe. This, in spite of the fact that the strange surface of Itokawa, characterized by a small amount of regolith and by the presence of many boulders, looks very different with respect to the surface of other larger asteroids visited in the past by other space missions.

Recently, however, Cellino et al. (2006) discovered that asteroid (234) Barbara exhibits a phase-polarization curve that is absolutely anomalous due to a strongly negative polarization at phases larger than $20^{\circ}$. Cellino et al. proposed a few preliminary conjectures to explain this behavior, but the possible reasons of the peculiar polarimetric properties of this asteroid remained essentially unknown and finding a plausible explanation of their observations is theoretically challenging since this object does not have any known analogous among other atmosphereless bodies of our Solar System, for which we have available polarimetric data.

For a long time (234) Barbara has been taxonomically classified as a member of the big $\mathrm{S}$ taxonomic class (Tedesco et al. 1989; Tholen 1989). More recently, however, Bus \& Binzel (2002) pointed out that a number of objects previously classified as S-type can now be classified into separate classes. In particular, the K- and L-classes are characterized by a reddish spectral slope, followed at longer wavelengths by a slightly negative (K-class) to flat (L-class) trend. However, from the point of view of taxonomy, (234) Barbara belongs to a further subset of the L taxonomic class, characterized by a particularly reddish spectral trend. There are only 12 objects known to belong to this subclass, named Ld, and (234) Barbara is the largest of them (Bus \& Binzel 2002). As no polarimetric data were available for the other known Ld asteroids, we did not know whether the unusual polarimetric behavior of Barbara might be typical of this class.

In the present paper, we report polarimetric observations obtained during different polarimetric campaigns on asteroids (172) Baucis, (234) Barbara, (236) Honoria, (679) Pax, and (980) Anacostia, which are new cases of Barbara-like polarimetric behavior among main belt asteroids belonging to the K-, L-, and Ld-classes. In Sect. 2, we describe the observations and, in Sects. 3 and 4, we present and discuss our results.

\section{Observations}

We carried out observations during different observing runs between September 2005 and September 2007 at the $2.15 \mathrm{~m}$ telescope of Complejo Astronómico El Leoncito (CASLEO), San Juan, Argentina, using the Torino and CASPROF polarimeters. A full description of the Torino photopolarimeter can be found in Piirola (1988) and Scaltriti et al. (1989). Here we recall that this instrument allows for simultaneous measurement of polarization in five bands, using separate photomultipliers and a set of dichroic filters. CASPROF is a two-hole aperture polarimeter with rapid modulation provided by a rotating achromatic halfwave retarder and Wollaston prism polarizing beamsplitter. The complementary polarized beams are detected with photomultipliers operating in pulse-counting mode, and the acquisition and guiding are accomplished with a CCD camera viewing the sky surrounding the entrance aperture. Since the received signal is, in general, exceedingly low in bands other than $V$ and $R$ in both instruments, only data obtained in these two bands were considered. From the analysis of several standard stars, we found the instrumental polarization fairly constant and always below $0.1 \%$ for both instruments.

We observed the targets during runs some weeks apart to obtain measurements during the same apparition at different phase angles. In order to check the performances of the instruments, we observed a few bright asteroids for which polarimetric data were already available in the literature. In these cases, the observations always fit the previously-known phase-polarization curves of these objects.

Observing nights were generally assigned around the new Moon to minimize the contamination of sky polarization by moonlight when we used the CASPROF polarimeter. In all cases, we used the smallest diaphragm allowed by the observing conditions to minimize the contribution of sky background. Each night we observed a minimum of two zero-polarization standard stars and one high-polarization star to determine instrumental polarization. The standard stars data were obtained from Turnshek et al. (1990) and Gil-Hutton \& Benavidez (2003).

We observed the targets consecutively several times each night with individual exposure times long enough to reach acceptable signal-to-noise ratios (at least $90 \mathrm{~s}$ and $180 \mathrm{~s}$ for the Torino and CASPROF polarimeters, respectively). The measurements for each retarder position were coadded to improve the $\mathrm{S} / \mathrm{N}$ ratio and the measurement errors were evaluated assuming a Poisson distribution. After a correction for instrumental polarization, we obtained the Stokes parameters with reduction programs specially designed for each polarimeter, with some modifications to adapt the reduction to the specific needs of asteroid polarimetry, including the computation of the position angle of the scattering plane and the derivation of the $P_{r}$ parameter.

\section{Results}

We present new data for (234) Barbara itself, and for the asteroids (172) Baucis, (236) Honoria, (679) Pax, and (980) Anacostia, which have been found to exhibit a polarimetric behavior similar to that of Barbara during these campaigns. The asteroid name, date, observing band, total integration time in seconds, phase angle, position angle of the scattering plane, degree 
Table 1. Asteroids with Barbara-like polarimetric behavior observed with the CASPROF polarimeter.

\begin{tabular}{llcrrrrrrrr}
\hline \hline Asteroid & Date UT & Band & $\begin{array}{c}T_{\text {int }} \\
\mathrm{S}\end{array}$ & \multicolumn{1}{c}{$\begin{array}{c}\alpha \\
{ }^{\circ}\end{array}$} & $\begin{array}{c}\theta_{\odot} \\
{ }^{\circ}\end{array}$ & $\begin{array}{c}P \\
\%\end{array}$ & $\begin{array}{c}\sigma_{P} \\
\%\end{array}$ & $\begin{array}{r}\theta \\
{ }^{\circ}\end{array}$ & $\begin{array}{r}\sigma_{\theta} \\
{ }^{\circ}\end{array}$ & $\begin{array}{r}P_{r} \\
\%\end{array}$ \\
\hline 172 Baucis & 2007 Jul. 10 & $V$ & 1440 & 28.4 & 66.5 & 0.10 & 0.04 & 172.0 & 10.8 & 0.09 \\
& 2007 Jul. 11 & $V$ & 480 & 28.4 & 66.4 & 0.11 & 0.04 & 144.6 & 9.7 & 0.10 \\
& 2007 Aug. 13 & $V$ & 960 & 22.9 & 63.2 & 0.86 & 0.02 & 59.0 & 0.6 & -0.85 \\
& 2007 Sep. 10 & $V$ & 480 & 12.1 & 48.7 & 1.48 & 0.02 & 46.9 & 0.4 & -1.47 \\
234 Barbara & 2005 Sep. 04 & $V$ & 720 & 30.4 & 82.2 & 0.72 & 0.04 & 172.2 & 1.4 & 0.72 \\
236 Honoria & 2007 Jun. 15 & $V$ & 960 & 18.7 & 65.4 & 1.24 & 0.03 & 72.1 & 0.7 & -1.20 \\
& 2007 Jul. 10 & $V$ & 960 & 11.3 & 52.6 & 1.24 & 0.02 & 58.3 & 0.5 & -1.21 \\
& 2007 Jul. 11 & $V$ & 960 & 11.0 & 51.6 & 1.24 & 0.02 & 58.4 & 0.4 & -1.21 \\
& 2007 Aug. 13 & $V$ & 480 & 7.0 & 114.7 & 1.00 & 0.02 & 110.1 & 0.6 & -0.98 \\
& 2007 Sep. 10 & $V$ & 960 & 17.4 & 86.0 & 1.15 & 0.02 & 80.9 & 0.5 & -1.14 \\
980 Anacostia & 2007 Jul. 11 & $V$ & 480 & 6.7 & 57.9 & 0.98 & 0.02 & 63.5 & 0.4 & -0.96 \\
& 2007 Aug. 13 & $V$ & 480 & 10.8 & 100.5 & 1.26 & 0.01 & 97.5 & 0.3 & -1.25 \\
& 2007 Sep. 10 & $V$ & 960 & 21.0 & 89.1 & 0.81 & 0.02 & 85.0 & 0.7 & -0.81 \\
\hline
\end{tabular}

Table 2. Asteroids with Barbara-like polarimetric behavior observed with the Torino polarimeter.

\begin{tabular}{lcccccccccc}
\hline \hline Asteroid & Date UT & Band & $\begin{array}{c}T_{\text {int }} \\
\text { s }\end{array}$ & $\begin{array}{c}\alpha \\
{ }^{\circ}\end{array}$ & $\begin{array}{c}\theta_{\odot} \\
{ }^{\circ}\end{array}$ & $\begin{array}{c}P \\
\%\end{array}$ & $\begin{array}{c}\sigma_{P} \\
\%\end{array}$ & $\begin{array}{c}\theta \\
{ }^{\circ}\end{array}$ & $\begin{array}{r}\sigma_{\theta} \\
{ }^{\circ}\end{array}$ & $\begin{array}{c}P_{r} \\
\%\end{array}$ \\
\hline 234 Barbara & 2005 Sep. 28 & $V$ & 720 & 25.8 & 93.1 & 0.59 & 0.17 & 102.8 & 6.3 & -0.56 \\
& 2005 Sep. 28 & $R$ & 720 & 25.8 & 93.1 & 0.28 & 0.14 & 101.8 & 10.6 & -0.27 \\
& 2005 Sep. 29 & $V$ & 720 & 25.5 & 93.7 & 0.44 & 0.16 & 113.3 & 7.2 & -0.34 \\
& 2005 Sep. 29 & $R$ & 720 & 25.5 & 93.7 & 0.40 & 0.08 & 95.5 & 4.9 & -0.40 \\
& 2005 Sep. 30 & $V$ & 720 & 25.3 & 94.3 & 0.61 & 0.13 & 111.2 & 4.4 & -0.51 \\
& 2005 Sep. 30 & $R$ & 720 & 25.3 & 94.3 & 0.46 & 0.10 & 104.9 & 4.5 & -0.43 \\
& 2005 Oct. 01 & $V$ & 720 & 25.0 & 94.9 & 0.57 & 0.10 & 97.7 & 4.5 & -0.57 \\
& 2005 Oct. 01 & $R$ & 720 & 25.0 & 94.9 & 0.55 & 0.08 & 93.1 & 4.0 & -0.55 \\
& 2006 Feb. 05 & $V$ & 720 & 24.5 & 74.2 & 0.55 & 0.15 & 79.7 & 6.7 & -0.54 \\
& 2006 Feb. 05 & $R$ & 720 & 24.5 & 74.2 & 0.73 & 0.11 & 74.7 & 6.7 & -0.73 \\
& 2007 Mar. 15 & $V$ & 720 & 8.4 & 121.1 & 1.61 & 0.18 & 122.2 & 3.1 & -1.61 \\
& 2007 Mar. 15 & $R$ & 720 & 8.4 & 121.1 & 1.25 & 0.14 & 112.9 & 2.5 & -1.20 \\
& 2007 Sep. 03 & $V$ & 720 & 20.0 & 142.9 & 1.34 & 0.04 & 139.7 & 0.7 & -1.33 \\
& 2007 Sep. 03 Pax & $R$ & 720 & 20.0 & 142.9 & 1.35 & 0.04 & 141.7 & 0.7 & -1.35 \\
& 2007 Sep. 04 & $V$ & 720 & 20.1 & 144.7 & 1.35 & 0.06 & 146.7 & 1.2 & -1.35 \\
& 2007 Sep. 04 & $R$ & 720 & 20.1 & 144.7 & 1.36 & 0.02 & 144.3 & 0.5 & -1.36 \\
& 2007 Sep. 05 & $V$ & 720 & 20.2 & 146.6 & 1.35 & 0.07 & 147.4 & 1.4 & -1.35 \\
& 2007 Sep. 05 & $R$ & 720 & 20.2 & 146.6 & 1.26 & 0.03 & 144.9 & 0.6 & -1.26 \\
& 2007 Sep. 06 & $V$ & 720 & 20.3 & 148.4 & 1.29 & 0.07 & 146.2 & 1.4 & -1.28 \\
& 2007 Sep. 06 & $R$ & 720 & 20.3 & 148.4 & 1.34 & 0.03 & 146.2 & 0.7 & -1.34 \\
& 2007 Sep. 09 & $V$ & 720 & 20.7 & 153.8 & 1.14 & 0.04 & 152.6 & 0.9 & -1.14 \\
& 2007 Sep. 09 & $R$ & 720 & 20.7 & 153.8 & 1.22 & 0.03 & 153.9 & 0.7 & -1.22 \\
\hline
\end{tabular}

of linear polarization and its error, position angle in the equatorial reference frame and its error, and $P_{r}$ are shown in Tables 1 and 2.

Since normally the polarimetric observations obtained in $V$ and $R$ bands agree very well with each other, measurements for these objects in both bands are plotted together in Fig. 1, where the few measurements already available for these asteroids in the literature are also included: three points for (172) Baucis from Zellner \& Gradie (1976) and Lupishko (2006), and the $V$ - and $R$-band data for (234) Barbara from Cellino et al. (2005b). As a comparison, we also plotted polarimetric data of (12) Victoria, a typical L-class asteroid. Figure 1 shows that the polarimetric properties of the objects listed in Tables 1 and 2 exhibit remarkable differences with respect to the more usual properties of (12) Victoria. In spite of the fact that these asteroids belong to different taxonomic classes, from the polarimetric point of view they exhibit a very similar behavior and constitute a homogeneous sample. Since previous analysis of asteroidal phase-polarization curves showed that asteroids with similar surface properties have similar polarimetric behavior (Penttilä et al. 2005; Goidet-Devel et al. 1995;
Gil-Hutton 2007), it is possible to obtain polarimetric parameters for this peculiar group using all the observations for the five asteroids and the semi-empirical model of phase-polarization curves proposed by Piironen et al. (2000), M. Kaasalainen et al. (2001), S. Kaasalainen et al. (2001), and Muinonen et al. (2002a):

$P_{r}(\alpha)=A_{0}\left[\exp \left(-\frac{\alpha}{A_{1}}\right)-1\right]+A_{2} \alpha$

where $A_{0}, A_{1}$, and $A_{2}$ are constant coefficients. With this method, we found a minimum of the phase-polarization curve of $\left|P_{\min }\right|=$ $1.36 \pm 0.13 \%$ at $\alpha_{\min }=13.8^{\circ}$, a slope of the linear region of the phase-polarization curve of $h=0.183 \pm 0.007 \% /{ }^{\circ}$, and an inversion angle of $\alpha_{0}=28.4^{\circ}$. As a comparison, the values obtained for (12) Victoria are $\left|P_{\min }\right|=0.73 \pm 0.01 \%$ at $\alpha_{\min }=9.7^{\circ}$; $h=0.121 \pm 0.001 \% /^{\circ}$; and $\alpha_{0}=20.8^{\circ}$.

The asteroids (172) Baucis and (234) Barbara have enough measurements ( 7 and 22 observations, respectively) to calculate a phase-polarization curve for each one. Using the same approach as for the whole group of asteroids, the values obtained 


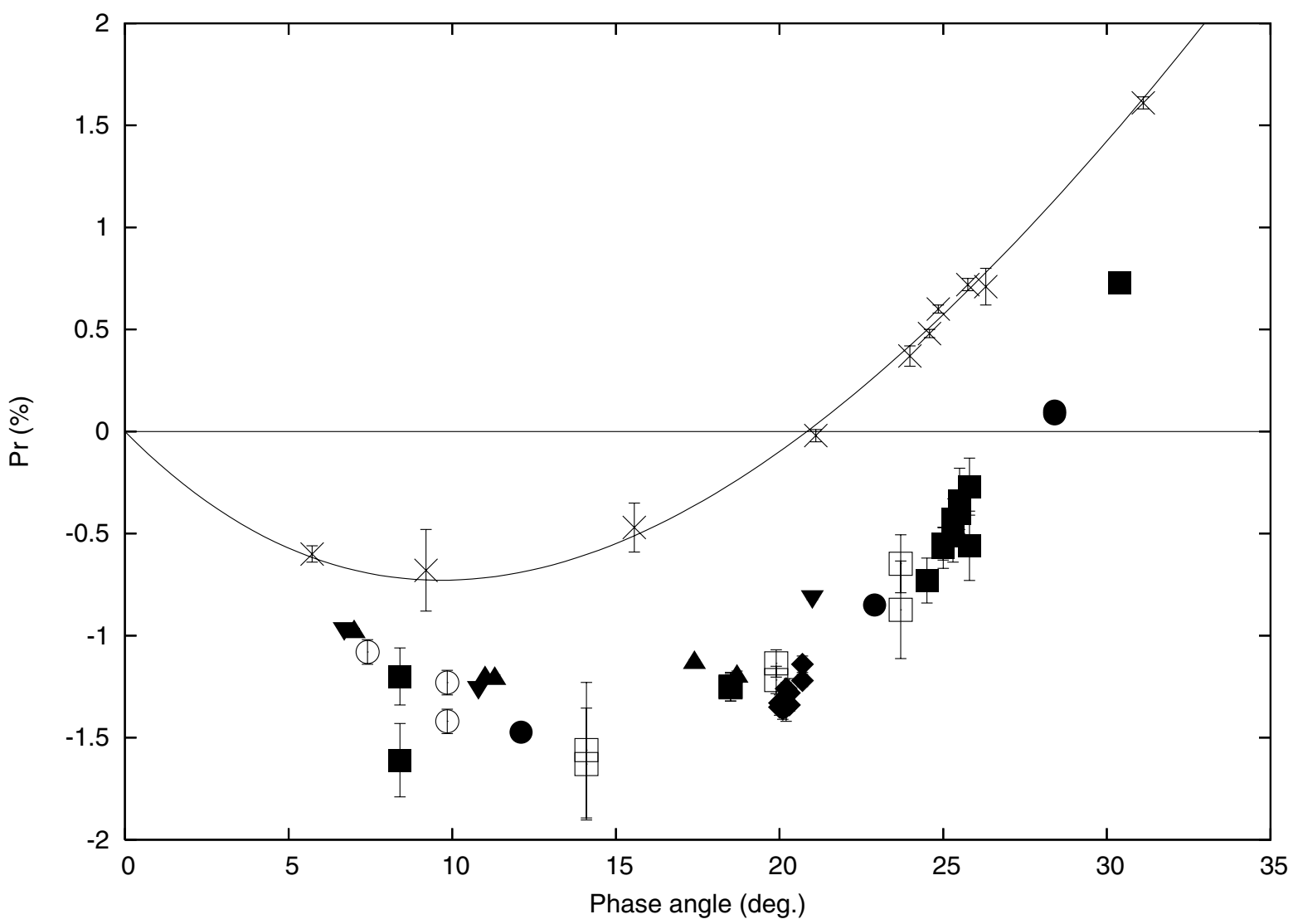

Fig. 1. Polarimetric observations of asteroids with Barbara-like polarimetric properties. Data for (172) Baucis are indicated by circles, for (234) Barbara by squares, for (236) Honoria by triangles, for (679) Pax by diamonds, and for (980) Anacostia by inverted triangles. Filled symbols indicate data taken during our present campaign. For a comparison, data for the L-class asteroid (12) Victoria are displayed by crosses, together with the corresponding fit of its phase-polarization curve.

for the polarimetric parameters that characterize the phasepolarization curve of these objects are $\left|P_{\min }\right|=1.48 \pm 0.03 \%$ at $\alpha_{\min }=13.6^{\circ}, h=0.200 \pm 0.002 \% /^{\circ}$, and $\alpha_{0}=28.0^{\circ}$ for Baucis; and $\left|P_{\min }\right|=1.52 \pm 0.08 \%$ at $\alpha_{\min }=13.2^{\circ}, h=$ $0.206 \pm 0.005 \% /{ }^{\circ}$, and $\alpha_{0}=27.3^{\circ}$ for Barbara. These values are similar to those found for the whole group.

It is possible to use these parameters to find the polarimetric albedo $p$ applying two empirical relations linking it with $h$ or $P_{\min }$. These relations are expressed by means of very simple mathematical forms:

$\log p(h)=C_{1} \log h+C_{2}$

$\log p\left(P_{\min }\right)=C_{3} \log P_{\min }+C_{4}$

where $C_{1}, C_{2}, C_{3}$, and $C_{4}$ are constants. In this paper we use the set of constants proposed by Cellino et al. (1999), namely: $C_{1}=-1.118 \pm 0.071, C_{2}=-1.779 \pm 0.062, C_{3}=-1.357 \pm 0.140$, and $C_{4}=-0.858 \pm 0.030$. Using these empirical relations with the polarimetric parameters found previously, we obtain $p(h)=0.11 \pm 0.02$ and $p\left(P_{\min }\right)=0.09 \pm 0.01$ for all the asteroids in the group, $p(h)=0.10 \pm 0.02$ and $p\left(P_{\min }\right)=0.08 \pm 0.01$ for (172) Baucis, and $p(h)=0.10 \pm 0.02$ and $p\left(P_{\min }\right)=0.08 \pm 0.01$ for (234) Barbara. It is clear, however, that the above albedo values have to be taken with some care because we are applying here the usual relations to find the albedo to objects which display an unusual polarimetric behavior, and we cannot be sure a priori that these relations also hold in this case.

\section{Discussion}

Since our polarization measurements for these objects have been obtained on different nights during different observing runs, and they agree well with each other with reasonably small error bars, we conclude that the observed behavior is real and represents new cases of asteroids exhibiting a strongly negative polarization at phases larger than $20^{\circ}$.

In Table 3, we list a summary of our current knowledge of the dynamical and physical parameters for these asteroids. They are objects distributed along the main belt, having typical sizes and albedos. The only physical parameter in common is that all of them have similar taxonomic classes: four asteroids belong to the L-class and the other one to the K-class. These taxonomic classes were proposed by Bus \& Binzel (2002) and are subsets of the old broad S-class defined by Tholen (1989). The differences between them are a change in the UV slope shortward of $0.75 \mu \mathrm{m}$ from moderately steep (K-class) to very steep (L-class), and a subtle change in the spectral slope longward of $0.75 \mu \mathrm{m}$. Since their sizes do not allow them to support a developed regolith of fine particles and the only agreement in their physical properties is their similar taxonomic class, the broad negative branch of their phase-polarization curves must be explained by a physical property of the asteroid surface, its composition, or a combination of both.

The first clue comes from the work of Zellner et al. (1977) on laboratory polarimetry of meteorites. These authors found peculiarly large values of the inversion angle for the carbonaceous chondrites, especially for the C3 samples, reaching $\alpha_{0}$ 
Table 3. Dynamical and physical properties of asteroids with Barbara-like polarimetric behavior.

\begin{tabular}{lrrrrrrrl}
\hline \hline Asteroid & \multicolumn{2}{c}{ Orbital elements $^{a}$} & \multicolumn{1}{c}{$\mathrm{H}^{b}$} & Rot. Period $^{c}$ & Size $^{d}$ & Geom. albedo $^{e}$ & Tax. Class $^{f}$ \\
& $\mathrm{a}(\mathrm{AU})$ & $\mathrm{e}$ & $\mathrm{i}(\mathrm{deg})$ & $(\mathrm{mag})$ & \multicolumn{1}{c}{$\mathrm{h})$} & \multicolumn{1}{c}{ (km) } & & \\
\hline 172 Baucis & 2.38 & 0.11 & 10.0 & 8.79 & 27.417 & 62.4 & $0.14 \pm 0.01$ & $\mathrm{~L}$ \\
234 Barbara & 2.39 & 0.24 & 15.4 & 9.02 & 26.500 & 43.8 & $0.23 \pm 0.01$ & $\mathrm{Ld}$ \\
236 Honoria & 2.80 & 0.19 & 7.7 & 8.18 & 12.333 & 86.2 & $0.13 \pm 0.01$ & $\mathrm{~L}$ \\
679 Pax & 2.59 & 0.31 & 24.4 & 9.01 & 8.452 & 51.5 & $0.17 \pm 0.02$ & $\mathrm{~K}$ \\
980 Anacostia & 2.74 & 0.20 & 15.9 & 7.85 & 20.117 & 86.2 & $0.17 \pm 0.01$ & $\mathrm{~L}$ \\
\hline
\end{tabular}

${ }^{a}$ From ftp://ftp. lowell. edu/pub/elgb/astorb.html. ${ }^{b}$ See Tedesco et al. (2002). ${ }^{c}$ From http://cfa-www.harvard.edu/iau/lists/ LightcurveDat.html. ${ }^{d}$ See Tedesco et al. (2002). ${ }^{e}$ See Tedesco et al. (2002). ${ }^{f}$ See Bus \& Binzel (2002).

values of $25^{\circ}$ or larger with $P_{\min }$ values around $1.2-1.5 \%$, in good agreement with the polarimetric parameters observed for the asteroids in our sample. Later, Burbine et al. (1992) suggested that the asteroid (980) Anacostia, one of the objects we found with Barbara-like polarimetric properties, could be a fragment of a spinel-bearing body with $\mathrm{CO} 3 / \mathrm{CV} 3$ affinities; Burbine et al. (2001) found a compositional analogy between K-class asteroids and CO3/CV3 chondrites; and Sunshine et al. (2007) identified the asteroid (234) Barbara as an spinel-rich object similar to Anacostia. These results suggest that the asteroids with these unusual polarimetric properties are mineralogically related with the carbonaceous chondrites and this meteorite type could be used as an analog for the $\mathrm{K}$ - and L-classes.

How does the mineralogy of $\mathrm{CO} 3 / \mathrm{CV} 3$ chondrites produce this peculiar phase-polarization curve? The spinel is an aluminum-magnesium oxide mineral $\left(\mathrm{MgAl}_{2} \mathrm{O}_{4}\right)$ that commonly incorporates small, but spectrally important amounts of transition metal cations such as iron and chromium, usually present in inclusions within a dark matrix in these meteorites. However, even though spinel is an important accessory mineral in the $\mathrm{CO} 3 / \mathrm{CV} 3$ meteorites, its physical abundance seldom exceeds a few percent. In spite of the fact that it probably makes up only a small percentage of the surface assemblage in these asteroids, its spectral effect may be enhanced by the spinel presence in finegrained white inclusions in the asteroid regolith (Burbine et al. 1992). In an immature regolith composed primarily of coarsedgrained crystalline fragments of their substrates (McKay \& Basu 1983) with white inclusions in a dark matrix, the inclusion fragments consist of aggregates of numerous transparent crystals that scatter photons efficiently. The contribution of the white inclusions to the net flux at a given wavelength is proportional to their relative abundance multiplied by a factor equal to the ratio of the spectral albedos of the white inclusion and dark matrix at that wavelength, contributing disproportionately to the total reflectance of the surface (Burbine et al. 1992). It is important to mention that this effect has some ressemblance with the effect proposed by Moretti et al. $(2005,2006)$ and produced by the presence of nanostructured metal particles on asteroid surfaces. These authors found that metal phases, which are usually reported in chondrites, produce a reddening of the reflectance spectrum. Since the spectra of L- and Ld-classes objects are strongly reddened, it might be that the polarimetric behavior and the reddening are parts of a same effect.

Then, due to its analogy with $\mathrm{CO} 3 / \mathrm{CV} 3$ chondrites, it is highly probable that these polarimetric anomalous asteroids have coarsed-grained regoliths formed by dark particles, coming from the matrix of the substrate, mixed with fine-grained white inclusions. In this scenario, it is important to note that a mixture of two components with different geometric albedo produces a negative polarization branch significantly more pronounced, and also a larger inversion angle, than the angle observed for a compositionally homogeneous surface of equivalent albedo (Shkuratov et al. 1994). This could affect the value observed for $P_{\min }$, and perhaps also the slope $h$, making it difficult to obtain reliable polarimetric albedos using the empirical relations linking this parameter with $h$ or $P_{\min }$ (Eqs. (2) and (3)) which hold for the large majority of objects not exhibiting a Barbara-like behavior. In fact, the polarimetric albedos obtained in this paper are considerably smaller than those obtained by IRAS for these asteroids (Tedesco et al. 2002, and Table 3), which is consistent with an interpretation based on the proposed scenario.

Petrographic studies of chondrules and inclusions in the CV3 Allende meteorite (McPherson et al. 1988) reveal highlyrefractory minerals similar in composition to those predicted to have condensed early in a gas of solar composition (Grossman 1972). This led to the suggestion that such inclusions might be some of the oldest materials found in meteorites (Tilton 1988). If the scenario proposed here to explain the anomalous polarimetric properties observed in some asteroids is valid, these objects also have very old material in their substrate and then must be among the oldest accreted bodies in the Solar System. Since they are distributed along the main asteroid belt from 2.38 to $2.80 \mathrm{AU}$, they are not fragments of an unique parent body and could represent localized accretion events throughout the asteroid belt.

Acknowledgements. The authors wish to thank K. Muinonen for his review of the manuscript. R.G.H. thanks M. E. Varela for her helpful comments.

\section{References}

Belskaya, I. N., Shkuratov, Yu. G., Efimov, Yu. S., et al. 2005, Icarus, 178, 213 Burbine, T. H., Gaffey, M. J., \& Bell, J. F. 1992, Meteoritics, 27, 424

Burbine, T. H., Binzel, R. P., Bus, S. J., \& Clark, B. E. 2001, Meteoritics Plan. Sci., 36, 245

Bus, S. J., \& Binzel, R. P. 2002, Icarus, 158, 146

Cellino, A., Gil-Hutton, R., Tedesco, E. F., Di Martino, M., \& Brunini, A. 1999, Icarus, 138,129

Cellino, A., Yoshida, F., Anderlucci, E., et al. 2005a, Icarus, 179, 297

Cellino, A., Gil-Hutton, R., Di Martino, M., et al. 2005b, Icarus, 179, 304

Cellino, A., Belskaya, I. N., Bendjoya, Ph., et al. 2006, Icarus, 180, 565

Gault, D. E., Horz, F., Brownlee, D. E., \& Hartung, J. B. 1974, Proc. Lunar Sci. Conf., 5, 2365

Geake, J. E., \& Dollfus, A. 1986, MNRAS, 218, 75

Gil-Hutton, R. 2007, A\&A, 464, 1127

Gil-Hutton, R., \& Benavidez, P. 2003, MNRAS, 345, 97

Goidet-Devel, B., Renard, J. B., \& Levasseur-Regourd, A. C. 1995, Planet. Space Sci., 43, 779

Grossman, L. 1972, Geochim. Cosmochim. Acta, 36, 597

Kaasalainen, M., Torppa, J., \& mulnonen, K. 2001, Icarus, 153, 37

Kaasalainen, S., Muinonen, K., \& piironen, J. 2001, Appl. Opt., 41, 4416

Langevin, Y., \& Arnold, J. R. 1977, Ann. Rev. Earth Planet. Sci., 5, 449

Le Bertre, T., \& Zellner, B. 1980, Icarus, 43, 172

Lupishko, D. F. 2006, (Ed.), Asteroid Polarimetric Database V5.0. EAR-A-3RDR-APD-POLARIMETRY-V5.0. NASA Planetary Data System McKay, D. S., \& Basu, A. 1983, J. Geophys. Res. Supp., 89, B193 
McPherson, G. J., Wark, D. A., \& Armstrong, J. T. 1988, in Meteorites and the Early Solar System, ed. J. F. Kerridge, \& M. Shapley-Matthews (Tucson: Univ. of Arizona Press), 746

Moretti, P. F., Maras, A., Palomba, E., et al. 2005, ApJ, 634, L117

Moretti, P. F., Maras, A., Somma, F., \& Aloe, P. 2006, A\&A, 459, 245

Muinonen, K., Piironen, J., Kaasalainen, S., \& Cellino, A. 2002, Mem. Soc. Astron. Ital., 73, 716

Muinonen, K., Piironen, J., Shkuratov, Yu. G., Ovcharenko, A., \& Clark, B. E. 2002, in Asteroids III, ed. W. F. Bottke Jr., A. Cellino, P. Paolicchi, \& R. P. Binzel (Tucson: Univ. of Arizona Press), 123

Muinonen, K., Zubko, E., Tyynelä, J., Shkuratov, Yu. G., \& Videen, G. 2007, JQSRT, 106, 360

O’Keefe, J. D., \& Ahrens, T. J. 1977, Science, 198, 1249

Penttilä, A., Lumme, K., Hadamcik, E., \& Levasseur-Regourd, A.-C. 2005, A\&A, 432, 1081

Piirola, V. 1988, in Polarized Radiation of Circumstellar Origin, ed. G. V. Coyne et al. (Tucson: Univ. of Arizona Press), 735

Piironen, J., Muinonen, K., Keränen, S., Karttunen, H., \& Peltoniemi, J. 2000, in Advances in Global Change Research, ed. M. Verstraete, M. Menenti, \& J. Peltoniemi (Dordrecht: Kluwer Academic), 4, 219
Scaltriti, F., Piirola, V., Cellino, A., et al. 1989, Mem. Soc. Astron. Ital, 60, 243 Shkuratov, Yu. G., Muinonen, K., Bowell, E., et al. 1994, Earth, Moon and Planets, 65, 201

Sunshine, J. M., Connolly Jr., H. C., McCoy, T. J., Bus, S. J., \& La Croix, L. 2007, Lunar and Planetary Science XXXVIII, 1613

Tedesco, E. F., Williams, J. G., Matson, D. L., et al. 1989, in Asteroids II, ed. R. P. Binzel, T. Gehrels, \& M. S. Matthews (Tucson: Univ. of Arizona Press), 1151

Tedesco, E. F., Noah, P. V., Noah, M., \& Price, S. D. 2002, AJ, 123, 1056

Tholen, D. J. 1989, in Asteroids II, ed. R. P. Binzel, T. Gehrels, \& M. S. Matthews (Tucson: Univ. of Arizona Press), 1139

Tilton, G. R. 1988, in Meteorites and the Early Solar System, ed. J. F. Kerridge, \& M. Shapley-Matthews (Tucson: Univ. of Arizona Press), 259

Turnshek D. A., Bohlin, R. C., Williamson II, R. L., Lupie, O. L., \& Koornneef, J. 1990, AJ, 99, 1243-1261, 1344-1377

Tyynelä, J., Zubko, E., Videen, G., \& Muinonen, K. 2007, JQSRT, 106, 520

Zellner, B., \& Gradie, J. 1976, AJ, 81, 262

Zellner, B., Leake, M., Lebertre, T., Duseaux, M., \& Dollfus, A. 1977, Proc. Lunar Sci. Conf. 8th, 1091 